\title{
Demo: A Software-Defined Radio for Wireless Brain Implants Network
}

\author{
Haili Cai ${ }^{\dagger}$, \\ Mustafa Lokhandwala ${ }^{\dagger}$, \\ Joey Zhu \\ University of California, San Diego \\ La Jolla, CA, USA \\ \{h7cai, mzlokhan\}@eng.ucsd.edu
}

\author{
Chester Kilfoyle, Jihun Lee, \\ Lawrence Larson, \\ Arto Nurmikko, \\ Farah Laiwalla \\ Brown University, \\ Providence, RI, USA \\ farah_laiwalla@brown.edu
}

\author{
Vincent W. Leung \\ University of California, San Diego \\ La Jolla, CA, USA \\ vwleung@eng.ucsd.edu
}

\begin{abstract}
A novel Brain-Machine Interface (BMI) system based on a distributed network of implantable wireless sensors was proposed. Small CMOS "Neurograin" chips $\left(0.5 \times 0.5 \mathrm{~mm}^{2}\right)$ with on-chip antenna are designed to harvest near-field $\mathrm{RF}$ energy at $\sim \mathrm{GHz}$, and backscatter $10 \mathrm{Mbps}$ BPSK modulated data asynchronously and periodically. A "Skinpatch" software-defined radio (SDR) receiver is realized on a commercial USRP running GNU Radio programs. It down-converts the reflected waves from the Neurograins and performs data recovery. In this BMI prototype demonstration, 32 Neurograins will be wirelessly powered, while a Skinpatch USRP will recover their backscattered packets in real-time.
\end{abstract}

\section{Introduction}

Brain-Machine Interfaces (BMIs) based on wired, invasive neural sensors [1] had seen significant advancement, particularly its successfully transition into human clinical trials. Wireless, fully-implantable neural interface technologies [2] are the next "holy-grail" of BMI arena. To dramatically increase the density and the number of channels, an approach comprising CMOS microscale "Neurograin" chips was proposed [3]. The prototype system targets the surface of the brain, where the individual nodes are organized into a 2-D grid that freely floats in the cerebrospinal fluid (CSF) surrounding the

†Co-primary authors

This research was developed with funding from the Defense Advanced Research Projects Agency (DARPA), Contract No. N66001-17-C-4013.

Permission to make digital or hard copies of part or all of this work for personal or classroom use is granted without fee provided that copies are not made or distributed for profit or commercial advantage and that copies bear this notice and the full citation on the first page. Copyrights for third-party components of this work must be honored. For all other uses, contact the Owner/Author.

MobiCom'18, October 29-November 2, 2018, New Delhi, India

(c) 2018 Copyright is held by the owner/author(s).

ACM ISBN 978-1-4503-5903-0/18/10.

https://doi.org/10.1145/3241539.3267727 brain, as demonstrated in Figure 1. The chip contains a dedicated RF micro-antenna and all the analog front-end, networking, RF power harvesting and backscattering circuits. To overcome low electromagnetic coupling due to the small on-chip antenna, a relay coil (antenna) is introduced to concentrate the flux from the Tx antenna [4]. To the author's best knowledge, it was the first demonstration of a BMI wireless sensor network inherently scalable to beyond 100s of channels.

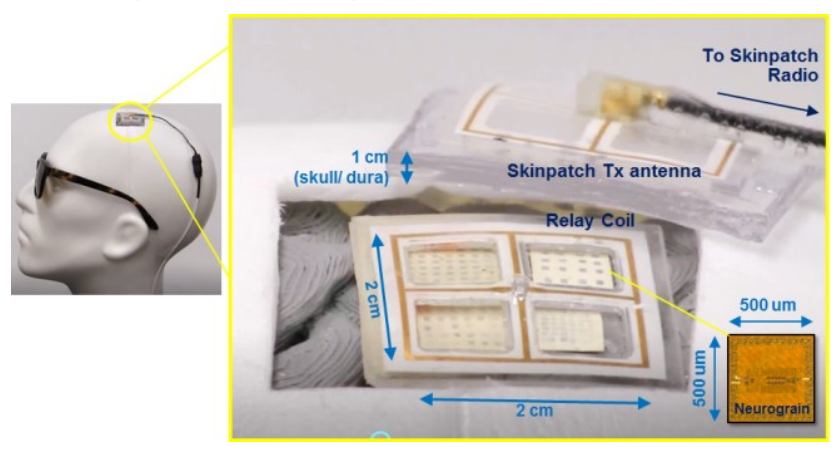

Figure 1: Cortical Surface Neurograin Sensor System.

Neurograin recorded data are backscattered to the "Skinpatch" demodulator. The recovered data are then channeled via neurocomputational processing units to the functional prosthetic devices. The intended full system data flow is depicted in Figure 2. The dotted box shows the demonstration described in this paper.

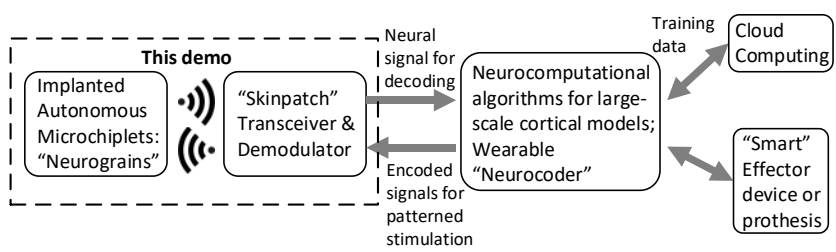

Figure 2: Overview of Neurograin system.

For the initial prototype, we have chosen to implement a 1000-channel system, with an overall system latency of 
$<100 \mathrm{~ms}$ (compatible with neural prosthetic applications). Design specifications such as data rates (10 Mbps), packet duration (100 $\mu \mathrm{s}$, or 1024 bits) and packet periodicty (100 $\mathrm{ms}$ data frame) are derived from these considerations.

\section{Neurograin and Skinpatch}

\subsection{CMOS Neurograin ASIC}

Figure 3 shows the block diagram of the prototype Neurograin ASIC. RF power at $\sim 1 \mathrm{GHz}$ is coupled through the on-chip coil (L) to the rectifier, which then produces a DC voltage to power all on-chip circuits. The oscillator free-runs at start-up. No locking mechanism is implemented for circuit simplicity and low power consumption. For initial testing purposes, we use a 31-bit linear feedback shift register (LFSR) to produce a fixed 10 Mbps digital pattern. This data stream is converted into a Manchester-coded BPSK-encoded signal, which toggles a switchable capacitor ( $\mathrm{C} 2)$, thereby modulating the reflected RF waves (backscatter) to establish wireless uplink communication [3]. Oscillator (clock) frequency is chosen at $30 \mathrm{MHz}$ to accommodate the backscattered spectrum.

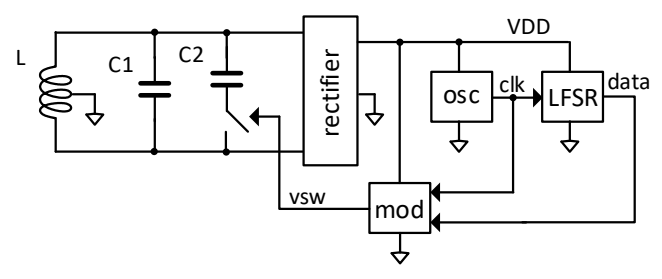

Figure 3: Neurograin test chip block diagram.

This chip provides an effective means to verify wireless power transfer and uplink communication performance. The deterministic nature of uplink data (LFSR bit pattern) allows for link fidelity validation through bit error rate (BER) computation from the measured received bits. To form an asynchronous network, digital logics are added to achieve "packetized" backscatter $(100 \mu$ s packets at $100 \mathrm{~ms}$ periodicity). Physically unclonable functions (PUFs) are built to randomize the backscatter start time per chip.

The Neurograin test chip has been designed and fabricated in TSMC $65 \mathrm{~nm}$ CMOS LP process. Figure 4(a) shows the chip photomicrograph. The outer coil (on-chip antenna) dimension is 500 by $500 \mu^{2}$. These chips will be spatially distributed in dense clusters and wirelessly interfaced with a single pair of $\mathrm{Tx}$ and relay antennae to form a multi-channel neurograin network. Figure 4(b) shows the 32-chip example employed in this demo. (a)

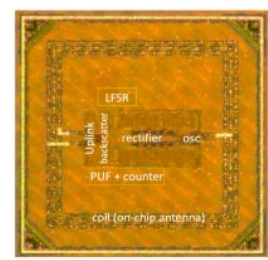

(b)

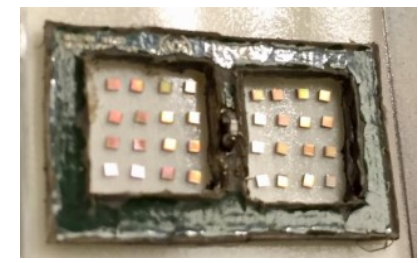

Figure 4: (a) Photomicrograph of the Neurograin test chip, and (b) a 32-chip network in a PCB relay antenna prototype.

\subsection{Skinpatch USRP SDR}

To receive the backscattered packets, the Skinpatch radio is realized by the commercial National Instrument USRP2900 [5]. This model covers up to $6 \mathrm{GHz}$ of RF frequency and $56 \mathrm{MHz}$ of instantaneous bandwidth. Baseband I/Q data, sampled at $45 \mathrm{Msps}$, are sent to a laptop for real-time signal processing. Skinpatch data demodulation and recovery are accomplished by "GNU Radio", a free software distributed under the GNU general public license. Users can realize the desired signal-processing functions by connecting a vast variety of pre-developed signal processing blocks (implemented in $\mathrm{C}++$ ) on a flowgraph.

Figure 5 shows the GNU Radio flowgraph for the Skinpatch receiver. "Power Squelch" detects the beginning and end of valid packets, defined by received backscattered power exceeding certain threshold. "Costas loop" performs frequency recovery. "Clock Recovery MM" performs timing recovery by using the real-time "number of samples per symbol ("sps")" information evaluated from the previous stage. This SDR receiver effectively combats the network asynchronicity due to the free-running Neurograin oscillators.

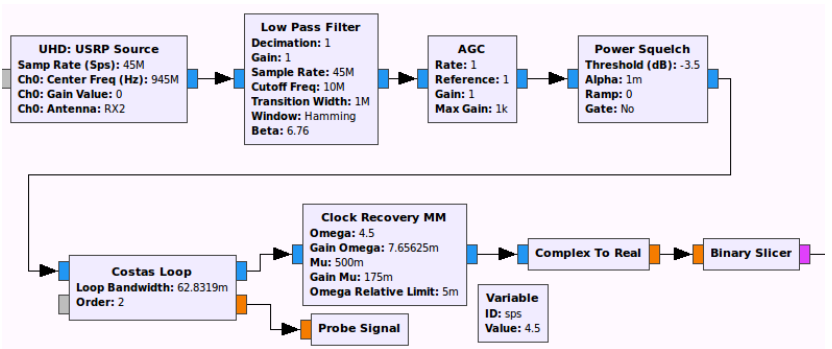

Figure 5: GNU Radio flowgraph of the Skinpatch receiver.

\section{Skinpatch Receiver Demo}

Figure 6 shows the setup for the wireless SkinpatchNeurograins demo. A $915 \mathrm{MHz} \mathrm{RF}$ tone is generated by the USRP transmitter. It is boosted by a RF amplifier to 27 $\mathrm{dBm}$ and sent to the Tx PCB antenna. 32 Neurograins, together with the relay antenna, are placed at $1 \mathrm{~cm}$ away, where they would be powered and backscattering 
packetized, 10 Mbps bit patterns. Picked up by the Tx antenna, the received signals are isolated by a microwave circulator, notch-filtered (to reject the leaked $\mathrm{Tx}$ tone), and sent to the USRP receiver. After down-conversion, the USRP sends the I/Q baseband data to the laptop through USB 3.0, where GNU Radio will perform real-time data recovery. Finally, decoded bits are sent to Matlab through TCP for evaluation and book keeping purposes.

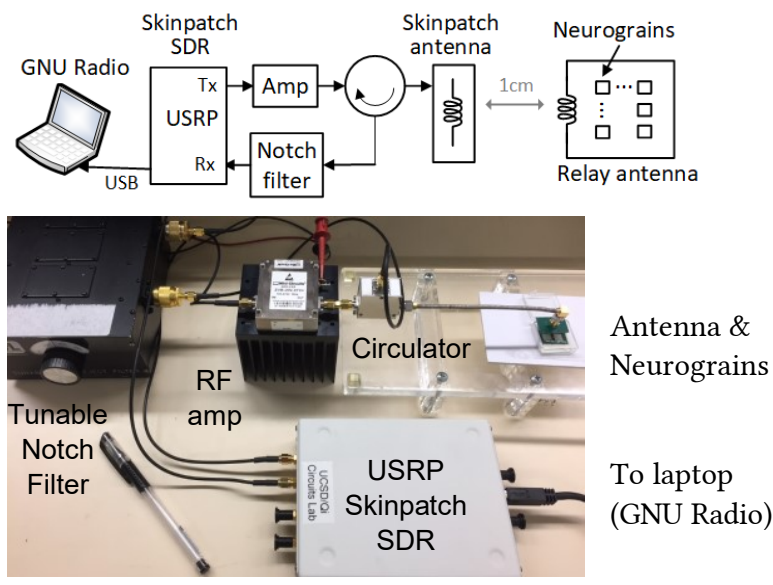

Figure 6: Skinpatch SDR and Neurograins demo.

We were able to detect and decode the $10 \mathrm{Mbps}$ data packets in real-time. Recall these packets are sent by different Neurograins at random time, and each of them could have slightly different power levels and data rates. In this 32-chip demo, $92 \%$ of the received packets demonstrate decent BER $(<0.3 \%$, while allowing for finite acquisition time). Figure 7 (a) shows a near-perfect received 1024-bit packet, where the repeating 31b LFSR pattern is displayed in a " $2 \mathrm{D}$ bitmap" for clarity. (a)
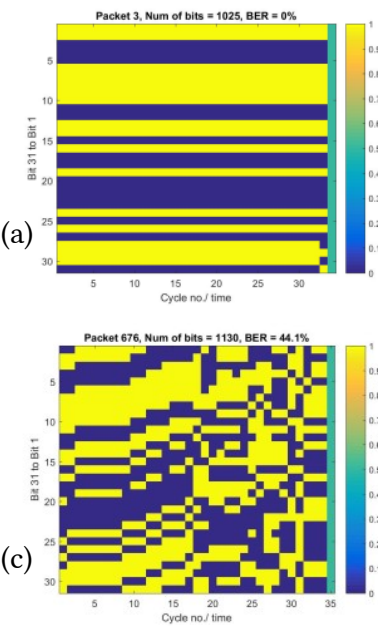

(b)

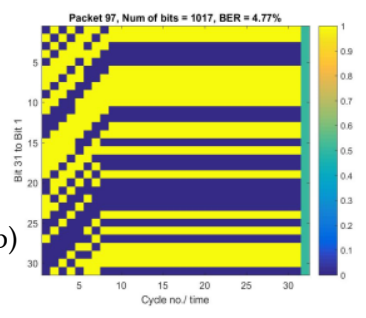

(d)

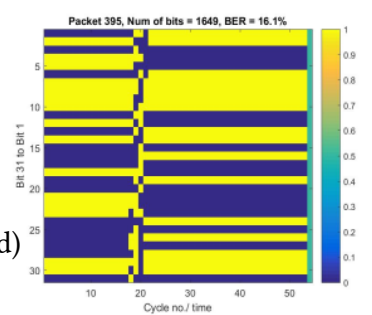

Figure 7: Decoded 2D bit-maps of received packets with (a) Good BER, (b) slow acquisition, (c) unstable recovery, and (d) collision.
About $4 \%$ of received packets exhibited prolonged acquisition time or unstable settling (Figure 7(b) and (c)). This is likely caused by chips located at spots with the weakest inductive coupling. Further optimization of certain GNU radio building blocks parameters (such as bandwidth, threshold) or test settings (such as Tx power and antenna tuning) could overcome these problems. Finally, about $4 \%$ of packets displayed "collision" (Figure $7(\mathrm{~d})$ ). This phenomenon is caused by randomized packet start time of each chip, and the measured collision rate agrees with the statistical prediction closely.

\section{Summary}

We have demonstrated an early prototype distributed sensor network for implantable BMI applications, where wireless power transfer and backscatter telemetry are verified over the targeted range. The relatively low-cost USRP and the straight-forward open-source GNU Radio program are proven adequate for real-time received packet demodulation and decoding.

\section{ACKNOWLEDGMENTS}

The authors acknowledge the contributions of Ms. Kayla Brosie of Northrop Grumman; Professor Peter Asbeck, Professor Patrick Mercier, and Mr. Jiannan Huang of UCSD; Dr. Steve Shellhammer of Qualcomm; Dr. David Durfee and Mr. Ryan Izant of Brown University.

\section{REFERENCES}

[1] L. R. Hochberg, M. D. Serruya, G. M. Friehs, J. A. Mukand, M. Saleh, A. H. Caplan, A. Branner, D. Chen, R.D. Penn, and J. P. Donoghue, "Neuronal ensemble control of prosthetic devices by a human with tetraplegia," Nature, 2006, vol. 442, pp. 164171.

[2] M. Yin, D. A. Borton, J. Aceros, W. R. Patterson, and A. V. Nurmikko, "A 100-channel hermetically sealed implantable device for chronic wireless neurosensing applications," IEEE Trans. on Biomedical Circuits and Systems, 2013, vol. 7(2), pp. 115-128.

[3] V. Leung, J. Lee, S. Li, S. Yu, C. Kilfoyle, L. Larson, A. Nurmikko, and F. Laiwalla, "A CMOS distributed sensor system for high-density wireless neural implants for brainmachine interface," to appear in 44th European Solid-State Circuits Conf (ESSCIRC), September 2018.

[4] J. Lee, F. Laiwalla, V. Leung, J. Jeong, C. Kilfoyle, L. Larson, A. Nurmikko, "Wireless transcutaneous powering of ensembles of chipscale sensors at microwaves frequencies," Int. Conf. IEEE Eng. Medicine and Biology Society (EMBS), July 2018.

[5] http://www.ni.com/pdf/manuals/374924c.pdf

[6] https://wiki.gnuradio.org/index.php/Main Page 